Idrissa Balde

Université Gaston Berger de Saint-Louis

(Sénégal)

\section{Une évaluation de la formation des inspecteurs de l'enseignement au Sénégal}

\author{
An evaluation of the training of education inspectors in Senegal
}

doi: $10.18162 / f p .2021 .598$

\section{$\mathrm{R}$ \\ ésumé}

L'évaluation de la formation des inspecteurs de l'enseignement cherche à répondre à une double interrogation sur la valeur de la formation et sur les compétences des inspecteurs dans un système éducatif à la recherche d'efficacité et de qualité. Ses référents sont les importantes missions de ces managers et les objectifs du plan de formation en vigueur. Les modèles théoriques visés sont

l'évaluation génératrice de développement professionnel et l'évaluation-conseil.

Les résultats de cette évaluation de formation dont la suggestion principale est le renforcement de la professionnalisation s'adressent aux acteurs de l'éducation et de la formation, notamment aux décideurs et aux formateurs.

Mots-clés Évaluation, formation, pilotage, professionnalisation, régulation.

Abstract

The benchmark for evaluating the training of education inspectors is the training plan implemented since the start of the 1999-2000 academic year. Beyond the strict application of Kirkpatrick's model which established a hierarchy and causal links between its four stages, this evaluation revealed others, with different objects and methods of achievement (evaluation of skills, evaluation of training system, selfevaluation, external evaluation). Strengthening professionalization, the main suggestion resulting from this evaluation refers to the evaluation that generates professional development for its purpose and to the evaluation-advice for its implementation.

Keywords

Assessment, training, management, professionalization, regulation.

\section{Introduction}

Dans les systèmes éducatifs, les missions des inspecteurs sont donc réparties en tâches d'administration pédagogique et tâches d'expertise administrative (Dickelé, 2005; Condette, 2017). Les activités de l'inspecteur de l'enseignement élémentaire sont référencées à des concepts qui illustrent leur statut de managers de l'éducation. Le contrôle, notamment le contrôle de conformité, est considéré comme son activité traditionnelle. Les inspecteurs sont chargés de la régulation interne (régulation intra-niveau de système) et de la régulation externe (relation du système éducatif et du système social) qui renferment chacune une régulation statique et une régulation dynamique (Berger, 1977). Par la régulation statique, ils cherchent à maintenir la cohérence du système et son homogénéité, d'assurer les transmissions, les décisions et les informations, de corriger les déformations possibles, de servir d'agent de liaison entre les différents niveaux hiérarchiques, mais aussi entre les différents agents à un même niveau. La régulation dynamique est le moyen de veiller à l'efficacité du système, d'introduire des innovations et des articulations entre des innovations sectorielles. Les inspecteurs de l'éducation sont au cœur du pilotage du système éducatif. Ils œuvrent dans le pilotage stratégique (définition des politiques et objectifs, affectation des ressources), dans le pilotage de gestion (répartition des tâches et des moyens en rapport avec les politiques et les objectifs du pilotage stratégique) et dans le pilotage opérationnel (procédures répétitives et décisions d'ajustement dans la réalisation des tâches).

Au Sénégal, les cadres investis de ces missions sont formés depuis 1962 à l'École Normale Supérieure (ENS) de Dakar qui est devenue en 2008 une structure de l'Université Cheikh Anta DIOP dénommée Faculté des Sciences et Technologies de l'Éducation et de la 
Formation (FASTEF). Dans le plan de formation en vigueur, il est indiqué : 1) la formation a précisément pour but de doter le futur inspecteur de compétences à la mesure des défis à relever dans le cadre de l'exercice de ses fonctions; 2) la première innovation consiste à réaliser un profil d'inspecteur polyvalent, c'est-à-dire capable d'intervenir à la fois au préscolaire et à l'élémentaire d'une part, et dans les secteurs formel et non formel d'autre part; 3) les structures et services d'éducation et de formation des secteurs formel et non formel sont mis à contribution de manière systématique; 4) les intervenants dans la formation sont des formateurs de l'ENS, des vacataires nationaux (consultants, experts, universitaires, chefs de services administratifs, responsables de structures).

Compte tenu des missions importantes de ces fonctionnaires de l'État dans le système éducatif qui est à la recherche d'efficacité et de qualité et au regard des objectifs du plan de formation en vigueur, cette formation mérite d'être évaluée. Cette activité vise la vérification des attentes en matière de profil et de compétences des inspecteurs pour répondre aux besoins de l'École sénégalaise. Elle cherche à répondre à une double interrogation portant sur la valeur de la formation et sur la capacité des inspecteurs à assumer et assurer les missions qui sont les leurs.

L'étude s'adosse l'évaluation génératrice de développement professionnel (Jorro, 2007) avec ses apports positifs :1) contribue à une clarification des systèmes de valeurs qui orientent l'activité professionnelle; 2) annonce une culture de la professionnalité dans le fait de mettre en dialogue le réel et le souhaitable de l'activité; 3) rend visible la complexité de l'activité professionnelle; 4) enclenche et accompagne le projet de professionnalisation; 5) interroge les interactions contexte-acteur-activité professionnels; 6) questionne le sens de la responsabilité; 7) légitime un processus de professionnalisation, notamment lors des certification. Un autre aspect de l'évaluation de la formation des inspecteurs est son ancrage dans l'évaluation-conseil (Jorro, 2007), la possibilité de dépasser le constat ou le bilan, constituant des arrêts sur image, certes instructifs, mais souvent sans lendemain. L'évaluation-conseil, en tant que le chaînon intermédiaire entre l'évaluation et la régulation, explore les perspectives d'ajustement jusqu’à influencer l'amplitude de la régulation.

Cette évaluation de formation se veut "une réflexion sur l'évolution des métiers et des situations professionnelles, sur la nécessaire prise en compte de la professionnalité [...] des cadres, sur la qualité des dispositifs de professionnalisation» (Jorro, 2007, p. 12). L'évaluation de la formation des inspecteurs est une «volonté de faire retour sur l'action éducative ou formative pour interroger la valeur de cet agir jusqu’à envisager la nécessité d'ajustements » (Forestier \& Thélot, 2007, cités par Jorro, 2007, p. 7).

L'évaluation de cette formation est aussi une évaluation de politique publique. Elle se justifie aisément dans un contexte de développement de la culture de l'évaluation par l'utilité d'évaluer les formations et l'obligation démocratique d'évaluer les politiques publiques. Pour toutes ces raisons, les résultats de cette évaluation de formation s'adressent aux acteurs de l'éducation et de la formation, notamment aux décideurs et aux formateurs.

La présente étude comprend un cadre conceptuel, qui est en réalité le cadre de référence théorique de l'étude, les présentations de la méthodologie, des résultats, de la discussion des résultats et de la conclusion. 


\section{Cadre conceptuel de l'étude}

Les concepts (formation, professionnalisation et évaluation) sont choisis pour les relations qu'ils entretiennent. En effet, si la finalité de la formation est la professionnalisation, l'évaluation demeure le moyen d'apprécier la validité et la fiabilité à la fois de la formation et de la professionnalisation.

La formation est la préparation à un métier, c'est-à-dire à la mise en œuvre de compétences dans une situation de travail. Cette activité de former un professionnel reste un critère important de l'employabilité dans les sociétés modernes. La formation entretient une relation privilégiée avec le travail, la conjoncture économique, avec le marché du travail. De nos jours, elle est devenue une préoccupation pour tous (individus, entreprises, administrations) dans la mesure où elle est apparue comme un facteur de développement de performance et/ou de compétitivité. La formation se décline souvent en formation initiale et en formation continue.

La fonction de la formation, que ce soit en formation initiale ou continue englobe plusieurs autres fonctions. Ces fonctions sont d'ordre politique (décider de la formation ou de la satisfaction du besoin de formation), technique (rendre faisable la demande, l'affiner et indiquer les outils et moyens nécessaires et la mise en œuvre de la formation) et didactique (confectionner et mettre en place les actions pédagogiques nécessaires à l'acquisition de nouvelles compétences).

La formation est la plupart du temps un concept qui est assez mal défini, tant elle peut prendre des formes variées adaptées à chaque demande, besoin ou contexte. Le point sur lequel s'accordent les différents manuels en psychologie du travail (Huteau, 2003; Arnold, 2005 ; Spector, 2006) concerne les étapes successives que devrait idéalement suivre une activité de formation : évaluation des besoins, définition d'objectifs, préparation, activité de formation et évaluation de ses effets. (Gilibert \& Gillet, 2010, p. 218)

L'action de formation est le processus mis en œuvre dans le cadre d'objectifs de formation définis par les décideurs pour permettre aux apprenants d'atteindre les objectifs pédagogiques fixés par les formateurs. L'apprenant est la personne qui participe à un processus d'acquisition de connaissances, de savoir-faire opératoires et éventuellement de savoir-faire relationnels (formation initiale) et de maintien et d'amélioration de ceux-ci (formation continue).

Pour aborder le concept de professionnalisation, il est utile de clarifier ses relations avec quelques mots de même famille (Jeffrey, 2016, p. 7). Les critères qui déterminent la professionnalité sont : 1) le développement du statut professionnel (identité, autonomie, autorité) et la reconnaissance sociale de la profession; 2) la détermination et la standardisation des savoirs, des pratiques et des formations; 3) le professionnalisme et l'éthique. Ces mêmes critères déterminent le passage d'un métier à une profession et le niveau de professionnalité confère le statut professionnel. Le professionnalisme permet de juger de la qualité d'une pratique accomplie dans le respect des personnes qui reçoivent les services et dans le respect de l'art de faire. La professionnalisation concerne, d'une part, le rehaussement de l'identité et du statut, et d'autre part, la standardisation ou la normalisation des savoirs, des pratiques et des formations.

La professionnalisation comme «fabrication» d'un professionnel par la formation et quête d'une légitimité plus grande des offres et pratiques de formation» (Wittorski, 2009) suppose une articulation plus étroite entre l'acte de travail et l'acte de formation : 
Il ne s'agit plus seulement de transmettre de façon déductive des contenus pratico-théoriques ou, au contraire, d'apprendre sur le tas mais d'intégrer dans un même mouvement l'action au travail, l'analyse de la pratique professionnelle et l'expérimentation de nouvelles façons de travailler. (Wittorski, 2009, p. 3)

Cette conception de la professionnalisation montre qu'il s'agit d'un processus (Bourdoncle, 1993; Wittorski, 2009; Roquet, 2012). «Les processus de professionnalisation des individus, des activités et des organisations renvoient à une pluralité de modes de professionnalisation à l'intérieur de groupes professionnels plus ou moins établis» (Bourdoncle 1993, cité par Roquet, 2012). Wittorski (2009) a identifié six voies de professionnalisation déterminées par des logiques : logique de l'action, logique de la réflexion et de l'action, logique de la réflexion sur l'action, logique de la réflexion pour l'action, logique de traduction culturelle par rapport à l'action et logique de l'intégration assimilation. Roquet (2012) décrit ce processus en trois niveaux : macro (construction historique et sociale de l'activité), méso (les dispositifs institutionnels de formation et de travail) et micro (le vécu du sujet).

La professionnalisation mène à la reconnaissance professionnelle. Elle débute en formation «sur ce que font les individus en situation de formation ou de travail, à partir de qualités attendues ou attribuées dans des dispositifs spécifiques, et la manière dont ils sont reconnus dans ces mêmes situations» (Jorro \&Wittorski, 2013).

La professionnalisation des acteurs constitue une priorité pour les pays qui valorisent l'éducation tout au long de la vie. Les mutations du monde du travail et les besoins d'adaptation des acteurs aux contextes évolutifs ont intensifié l'intérêt pour les questions de formation professionnalisante, de dispositif d'alternance, de validation des acquis de l'expérience, de démarches d'accompagnement à l'insertion professionnelle, de reconnaissance professionnelle. (Jorro, 2014)

Le concept d'évaluation, pour sa part, peut être défini à l'instar de Talbot \& Arrieu-Mutel (2012, p. 69) à partir de six caractéristiques principales : 1) l'évaluation est valeur ou plus exactement elle permet d'extraire de la valeur (ex. valuere), d'estimer une valeur; 2) l'évaluation est mesure, c'est-à-dire qu'elle permet la comparaison (à une norme explicite ou implicite) et le constat (un état des lieux dans le sens d'une tentative d'objectivation); 3) l'évaluation est sens, sens comme signification (de la valeur attribuée) et sens comme direction (pour l'évaluation formative par exemple); 4) l'évaluation permet l'évolution en tant que dynamique. Il s'agit d'une activité d'intervention et d'accompagnement et d'une activité de conseil au sens de délibérer et de tenir conseil ou d'aider à décider et de donner conseil; 5) l'évaluation diffère du contrôle qui est un processus sanctionnant et clôturant; 6) l'évaluation est différente du jugement qui s'intéresse aux sujets.

L'évaluation est un processus qui se manifeste dans la confrontation d'un référent à un référentiel. Deux courants illustrent ce processus.

Le premier consiste à affirmer que l'évaluation est un processus conduisant à produire une appréciation ou un jugement (Hadji, 1989; Allal, 2007, cités par De Ketele, 2011, p. 90). Le second, auquel nous nous rattachons, estime que si le processus évaluatif comprend des démarches d'appréciation et de jugement, il a pour fin de fonder une prise de décision pertinente (ce qui répond à la question : «est-ce que je ne me trompe pas de...?»), valide (ce qui répond à la question: "ce que j'évalue réellement correspond-il bien à ce que je déclare évaluer?») et fiable (ce qui répond à la question : «la décision produite est-elle indépendante de l'évaluateur et des circonstances?»). (De Ketele, 2011, p. 90) 
La relation entre la formation et l'évaluation se trouve être la nécessité d'évaluer souhaitée par la communauté professionnelle des formateurs, des responsables des ressources humaines, les clients internes de la formation (dirigeants et managers) et les usagers directs que sont les participants aux actions de formation. Lévaluation des formations dépasse la collecte des preuves de la satisfaction des stagiaires et devient un outil indispensable pour asseoir durablement les effets bénéfiques d'une prestation de formation, les optimiser, démontrer et renforcer l'utilité réelle de la formation (Gilibert \& Gillet, 2010, p. 221). La multiplication des formations s'accommode avec la culture de l'évaluation imposée par le développement des pratiques démocratiques (la nécessité de rendre des comptes), l'attitude gestionnaire ou l'attitude de management dans la plupart des pratiques (phénomène de type organisationnel qui fait triompher les systèmes de gestion sur les systèmes de croissance des moyens) et la perte de légitimité sociale des grands secteurs de notre vie collective (demande constamment une nouvelle opération de légitimation) (Bonniol \& Vial, 2006).

Jorro $(2007,2015)$ évoque trois autres fonctions de l'évaluation en lien avec la formation. L'auteure présente tour à tour l'évaluation-conseil, l'évaluation génératrice de développement professionnel et l'évaluation qui mène à la reconnaissance professionnelle en formation. Lévaluation-conseil se situe entre l'évaluation et la régulation, à un endroit où sont définies des perspectives nouvelles et surgissent des possibilités qui serviront à justifier la faisabilité, la pertinence et l'efficience. En formation professionnelle, «en valorisant son projet d'action, en lui donnant l'envie d'aller au-delà de ce qu'il a fait jusqu'alors, les processus de conseil produisent de la réassurance et sollicitent l'estime de soi » (Jorro, 2007, p. 8). Dans l'évaluation des dispositifs de formation, l'évaluation-conseil consiste à proposer une interprétation qui saisit les points d'appui et les zones de fragilité, qui amplifie des dimensions encore trop discrètes et qui dégage l'impensé d'un système. «En établissant un lien entre le développement professionnel et l'évaluation, nous voulons mettre en évidence l'importance de la fonction critique dans la construction de la professionnalité des acteurs». (Jorro, 2007, p. 1) En effet, les apprentissages professionnels impliquent à des moments divers des processus d'évaluation (appropriation de compétences, incorporation de gestes professionnels, acquisition d'une culture de métier, etc.). L'évaluation est aussi à l'origine de développement chez les professionnels qui apprennent alors à mettre en œuvre une «culture de l'action à venir» (Jorro, 2007) en dépassant le seuil de l'évocation d'une action vécue pour franchir celui de l'anticipation de l'action. Jorro (2015) dénonce l'importance accordée à la mesure dans l'évaluation dans la formation des adultes, comme si seuls les résultats comptaient. La problématique de la reconnaissance accorde une place aux processus de transformation des acteurs dans la temporalité de la formation. Elle permet de saisir le positionnement des acteurs dans leurs processus de transformation dans les environnements de formation et de travail (Jorro, 2015, p. 41).

\section{Méthodologie}

L'évaluation de la formation des inspecteurs se propose de vérifier les attentes formulées dans le plan de formation en matière de profil et les compétences des inspecteurs au contact de la réalité du terrain et des besoins de l'École sénégalaise. 
Les participants à cette étude sont les inspecteurs entrés en service dans la période comprise entre les années 2000 et 2008 et les inspecteurs, chefs des services qui les ont accueillis à l'issue de leurs formations. La première cible comprend trois-cent-un (301) inspecteurs dont 285 hommes et 16 femmes (tableau 1). La seconde comprend les inspecteurs d'académie (IA), chefs de services régionaux, les inspecteurs de l'éducation et de la formation (IEF), chefs de services départementaux et les directeurs de centres régionaux de formation des personnels de l'éducation (CRFPE).

\section{Tableau 1}

Population ciblée d'inspecteurs

\begin{tabular}{|c|c|c|c|}
\multicolumn{1}{|c}{ Entrée dans le corps } & Total & Hommes & Femmes \\
\hline 2000 & 37 & 33 & 4 \\
\hline 2001 & 36 & 34 & 2 \\
\hline 2002 & 28 & 26 & 0 \\
\hline 2003 & 24 & 24 & 4 \\
\hline 2004 & 31 & 27 & 1 \\
\hline 2005 & 45 & 44 & 1 \\
\hline 2006 & 33 & 32 & 1 \\
\hline 2007 & 32 & 31 & 1 \\
\hline 2008 & 35 & 34 & 16 \\
\hline Total & 301 & 285 & \\
\hline
\end{tabular}

Source : Ministère de l'Éducation nationale

Lobjectif au départ était d'observer toute la population issue de cette formation. À l'arrivée, si toutes les promotions ont été représentées dans cette enquête, soixante-cinq (65) nouveaux inspecteurs ont répondu au questionnaire administré (tableau 2). Sept IA, IA, seize IEF, deux directeurs de CRFPE qui ont accueilli les inspecteurs sortant de formation ont participé à cette évaluation.

Tableau 2

Inspecteurs interrogés

Année d'entrée dans le corps

Effectif de la promotion

Nombre interrogé

\begin{tabular}{|c|c|c|}
\hline 2000 & 37 & 2 \\
\hline 2001 & 36 & 3 \\
\hline 2002 & 28 & 7 \\
\hline 2003 & 24 & 3 \\
\hline 2004 & 31 & 6 \\
\hline 2005 & 45 & 12 \\
\hline 2006 & 33 & 12 \\
\hline 2007 & 32 & 8 \\
\hline 2008 & 35 & 10 \\
\hline
\end{tabular}


Le profil sociodémographique en début de formation des nouveaux inspecteurs est ainsi reconstitué. Il y a plus d'hommes que de femmes (90,76 \% et 9,23 \%). Cette situation se confirme dans les promotions considérées (tableau 3). Les diplômes universitaires vont du baccalauréat (plus de $86 \%$ ) aux diplômes du second cycle (plus de 50 \%) en passant par les diplômes du premier cycle (35,38 \%). Les diplômes sont obtenus dans des disciplines variées (anglais, philosophie, lettres modernes, géographie, histoire, sociologie, sciences économiques, droit, biologie végétale, etc.). Dans les diplômes professionnels, il y a des diplômes d'instituteurs ou assimilés (plus de $78 \%$ ) dont des titulaires du certificat d'aptitude pédagogique $(63,07 \%)$ et du certificat d'aptitude à l'éducation préscolaire $(15,38 \%)$; des diplômes de professeurs de collège $(12,30 \%)$ dont des titulaires du certificat d'aptitude à l'enseignement dans les collèges d'enseignement moyen $(6,15 \%)$ et du certificat d'aptitude à l'enseignement moyen $(6,15 \%)$; des diplômes de professeurs de l'enseignement secondaire, des titulaires du certificat d'aptitude à l'enseignement secondaire (15,38 \%) et enfin des inspecteurs adjoints, titulaires du certificat d'aptitude aux fonctions d'inspecteurs adjoints de l'enseignement élémentaire (7,69\%). Les personnes interrogées proviennent (le dernier lieu de service avant la formation) des classes de l'enseignement élémentaire $(66,15 \%)$, des collèges et lycées $(26,15 \%)$ et de l'administration scolaire $(7,69 \%)$.

Divers outils d'investigation ont été utilisés pour la collecte de données (recherche documentaire, entretien questionnaires) avec des cibles variées. Les questionnaires restent le système d'information principal de notre étude.

\section{Tableau 3}

Outils d'investigation utilisés

\begin{tabular}{|c|c|c|}
\hline Outils & Cibles & Informations principales recherchées \\
\hline $\begin{array}{l}\text { Recherche } \\
\text { documentaire }\end{array}$ & $\begin{array}{l}\text { Ouvrages, revues, } \\
\text { thèses, rapports, plan } \\
\text { de formation }\end{array}$ & $\begin{array}{l}\text { Tout sur la profession d'inspecteur (histoire, missions, pratiques et leur } \\
\text { évolution }\end{array}$ \\
\hline Guide d'entretien & $\begin{array}{l}\text { Promotions de } 1962 \text { et } \\
\text { de } 1968\end{array}$ & Histoire de la formation des inspecteurs au Sénégal \\
\hline \multirow[t]{2}{*}{ Questionnaires } & Nouveaux inspecteurs & $\begin{array}{l}\text { Profil sociodémographique et professionnel, choix de l'inspection (images, } \\
\text { perceptions, influences), recueil des appréciations (apprentissages, } \\
\text { compétences, difficultés rencontrées) et proposition d'amélioration de la } \\
\text { formation }\end{array}$ \\
\hline & $\begin{array}{l}\text { Supérieurs } \\
\text { hiérarchiques }\end{array}$ & $\begin{array}{l}\text { Compétences attendues, Compétences décelées et proposition } \\
\text { d'amélioration de la formation }\end{array}$ \\
\hline
\end{tabular}

Le modèle d'évaluation utilisé est celui de Kirkpatrick avec quatre niveaux d'évaluation (les mesures des réactions, les mesures des apprentissages, l'observation des comportements et les résultats). Les réactions portent sur la perception de l'inspection scolaire avant la formation (images, motivations et sources de motivation), l'évaluation d'intrants et de l'environnement de la formation. La mesure de l'apprentissage est réalisée en trois mesures : la mesure des enseignements théoriques, la mesure des stages et la mesure de la stratégie de mise en œuvre de la formation. Dans la première mesure, 
l'évaluation de l'emploi du temps, l'intérêt pour les enseignements dispensés et pour les domaines de formation sont les opérations réalisées. Ensuite, l'organisation, l'encadrement et l'exploitation à des fins pédagogiques des stages ont été appréciés. La mise en œuvre de quelques principes retenus dans l'organisation de la formation est aussi mesurée : 1) l'ouverture de la formation aux structures et services d'éducation et de formation, des secteurs formel et non formel et aux interventions éventuelles de leurs experts dans la formation; 2) le renforcement des formateurs titulaires par des vacataires nationaux; 3 ) la formation d'un inspecteur de l'enseignement polyvalent, capable d'intervenir à la fois au préscolaire et à l'élémentaire d'une part, et dans les secteurs formel et non formel d'autre part. Les compétences attendues de la formation sont ainsi détaillées : 1) administration et de gestion des services centraux et des structures décentralisées du Ministère de l'Éducation nationale;2) formation initiale et continuée des maîtres de l'éducation préscolaire et de l'élémentaire des secteurs formel et non formel, public et privé; 3) supervision de l'organisation scolaire et des activités éducatives; 4) consultation pour les décideurs en matière d'éducation; 5) promotion du développement du réseau éducatif national; 6) contribution par la recherche, l'évaluation et l'innovation à l'amélioration de la qualité de l'éducation. Les compétences acquises et non acquises sont détectées à partir des activités. L'appréciation des résultats de la formation reste liée aux autres étapes de l'évaluation et à la confrontation des objectifs fixés avant la formation et les transferts de compétence dans les activités de terrain.

Les informations collectées ont été analysées de manière statistique. Pour chaque question, le nombre de réponses est recensé. Ensuite, les effectifs affectés aux valeurs sont transformés souvent en pourcentage afin de procéder, au besoin, à des classifications ou à déterminer le point de vue dominant ou l'information principale. Des regroupements de réponses ont été effectués pour déterminer, par exemple, les satisfactions (très satisfait et assez satisfait) et les insatisfactions (pas satisfait et pas du tout satisfait). Les compétences non acquises sont déterminées par le classement des compétences affectées d'effectifs importants en matière de difficultés rencontrées et des compétences non trouvées par les supérieurs hiérarchiques.

\section{Résultats}

\section{La mesure des réactions}

En début de formation, les élèves-inspecteurs percevaient l'inspection comme une nouvelle carrière dans la continuité de leur statut d'enseignant et afin d'aider les enseignants à améliorer leurs pratiques (grille 1).

Grille 1 : Recueil des perceptions du métier d'inspecteur

\begin{tabular}{|l|l|} 
Réponses proposées & \multicolumn{1}{l|}{ Pourcentage } \\
\hline C'est un métier dans la continuité des activités d'enseignement & 41,5 \\
\hline Un métier susceptible de faire progresser les pratiques des enseignants & 41,5 \\
\hline Un métier complexe avec la dimension de représentant de l'État auprès des différents partenaires & 9,2 \\
\hline Une représentation très floue & 6,1 \\
\hline
\end{tabular}

8 - Formation et profession 29(2), 2021 
Les principales motivations à intégrer le corps des inspecteurs (grille 2) sont, d'une part, l'aboutissement de plan de carrière et d'autre part, le souci de ne pas tomber dans un immobilisme professionnel.

Grille 2 : Recueil des motivations des élèves-inspecteurs

\begin{tabular}{|l|l|}
\begin{tabular}{ll} 
Réponses proposées & Pourcentage \\
\hline L'aboutissement logique de carrière au sein de l'Éducation nationale & 41,5 \\
\hline Le souci de ne pas tomber dans la routine enseignante & 18,4 \\
\hline Un intérêt "intellectuel": "avoir une hauteur de vue du système éducatif" & 10,7 \\
\hline Le désir d'avoir une fonction d'encadrement, de manager & 7,6 \\
\hline Un désir d'apprendre des choses nouvelles & 6,1 \\
\hline Promotion sociale & 6,1 \\
\hline
\end{tabular}
\end{tabular}

Plusieurs candidats à inspectorat (60,0 \%) ont subi une influence, venant principalement d'un inspecteur en activité (grille 3).

Grille 3 : Origines des influences subies

\begin{tabular}{|c|c|}
\hline Réponses obtenues & Pourcentage \\
\hline D'un inspecteur dans l'exercice de ses fonctions & 41,5 \\
\hline De collègues de travail & 9,2 \\
\hline De membres de la famille & 9,2 \\
\hline
\end{tabular}

Parmi les sources de forte influence venant d'un inspecteur, l'inspecteur rencontré dans le cadre de la pratique de la profession occupe la dernière place $(9,2 \%)$, tandis que l'inspecteur confident est mieux suivi $(20,0 \%)$.

Grille 4 : Caractéristiques de l'inspecteur influenceur

\begin{tabular}{|l|l|}
\hline Réponses obtenues & Pourcentage \\
\hline Tout part de l'inspecteur que l'enseignant connaît & 20 \\
\hline D'un inspecteur charismatique & 12,3 \\
\hline D'un inspecteur rencontré dans le cadre de la pratique de la profession & $9,23$. \\
\hline
\end{tabular}

Les satisfactions portent sur la durée de la formation $(67,6 \%)$, sur les méthodes pédagogiques $(66,1)$, sur le contenu de la formation $(66,1 \%)$, sur les ressources documentaires $(55,3 \%)$, sur le rythme de la formation $(52,3 \%)$ et sur la disponibilité des correspondants de stage $(50,7 \%)$. Les anciens stagiaires ne sont pas satisfaits du suivi individualisé par les formateurs (72,3\%), des ressources informatiques (56,9\%), de la disponibilité de l'équipe de direction (53,8 \%), de l'alternance théorie/pratique (43,0 \%), du rythme de la formation (41,5\%), de l'accueil et disponibilité des autres personnels (41,5\%), de la disponibilité des formateurs associés (40,0\%). 
Les relations entre les élèves-inspecteurs et les formateurs (suivi individualisé par les formateurs) recueillent le plus d'insatisfactions. Parmi les justificatifs avancés, il y a les profils inadéquats de certains formateurs, leur manque de motivation, leurs absences, les cours non menés à terme, les cours non dispensés, l'organisation des cours en tronc en commun (en français, pénalisant ainsi les élèves-inspecteurs de langue arabe) et l'absence de la mise en œuvre de la polyvalence dans les enseignements.

De l'environnement de la formation, les élèves-inspecteurs sont fiers de la bibliothèque $(58,4 \%)$. Ils ne sont pas pour l'hébergement $(92,3 \%)$, les locaux $(78,4 \%)$ et la restauration (80 \%). La délocalisation du lieu de la formation est la solution proposée pour rehausser son prestige et améliorer les conditions de vie et d'étude.

\section{La mesure de l'apprentissage}

L'emploi du temps est jugé chargé à 84,6\%, dont 52,3\% (chargé) et 32,3\% (peu chargé). Les enseignements les plus utiles sont : Psychologie, Théories de l'apprentissage, Didactique des disciplines, Mesure et évaluation en éducation, Administration, Législation, Techniques d'observation et d'analyse des activités, Management, Théories de l'éducation et Planification de l'éducation. Les enseignements considérés comme complémentaires des enseignements les plus utiles sont : Animation pédagogique, Initiation à l'informatique, Méthodes de recherche en éducation, Techniques de communication, Méthodologie du travail intellectuel, Initiation à la statistique et Ingénierie de la formation. Finalement, les enseignements les moins utiles sont : Économie de l'éducation, Épistémologie générale, Psychologie sociale, Andragogie, Techniques littéraires, Didactique des langues nationales et linguistique générale et appliquée. Les quatre domaines de compétences sont aussi classés dans l'ordre suivant : Éthique et responsabilité, Pratique professionnelle, Recherche et innovation, Communication et partenariat.

Pour ce qui est des modalités d'évaluation, le recueil des réponses collectées indique que le travail de groupe est privilégié.

Grille 5 : Appréciation des modalités d'évaluation en formation

\begin{tabular}{|l|l|}
\hline Réponses proposées & Pourcentage \\
\hline Incite au travail individuel & 15,3 \\
\hline Incite au travail de groupe & 63 \\
\hline N'exige pas d'effort soutenu & 7,6 \\
\hline Pas rigoureux & 10,7 \\
\hline Nese prononce pas & 3 \\
\hline
\end{tabular}

Une grande majorité des personnes interrogées $(66,1 \%)$ estime que les stages sont insuffisants dont $40 \%$, très insuffisants de par leur nombre, leur durée, la période de leur organisation et de par les lieux de stage. Elles justifient cette réponse par l'importance des stages dans la formation. L'encadrement des stages est défaillant $(69,2 \%)$ dont $29,2 \%$ d'avis passable et $40 \%$ pour pas d'encadrement du tout. Les rapports de stage ne sont jamais exploités (63 réponses sur 65 possibles). 
L'ouverture de la formation à plusieurs intervenants n'est pas effective. Les structures utilisatrices des inspecteurs et l'expertise extérieure ne sont pas associées à la formation. Les rares vacataires qui interviennent dans la formation font de la FASTEF « un dépotoir de hauts fonctionnaires de l'éducation déchus, sans le niveau requis encore moins la compétence $»^{1}$. «L'équipe est un club d'amis ouvert à des inspecteurs retraités ou en service dans l'administration ${ }^{2} »$.

La polyvalence souhaitée de l'inspecteur dans l'enseignement élémentaire et dans l'éducation préscolaire, d'une part, et d'autre part entre l'éducation formelle et l'éducation non formelle, n'est pas obtenue. Aucun enseignement n'est prévu dans l'emploi du temps pour le préscolaire et le non formel.

\section{Observation des comportements}

Le classement des activités des inspecteurs sur le terrain est établi par la sommation des citations des personnes interrogées. Les activités quotidiennes des nouveaux inspecteurs sont dans l'ordre: 1) inspection d'enseignants; 2) formation continue des enseignants; 3) inspection de direction; 4) formation initiale d'enseignants; 5) conduite de réunion; 6) communication écrite et orale; 7) management d'équipe; 8) expertise en éducation;9) conception de matériel didactique;10) évaluation du système éducatif. Au regard des compétences visées par la formation, les compétences acquises sont la supervision pédagogique $(78,4 \%)$ et la formation continue des maîtres $(69,2 \%)$. Celles qui restent à acquérir sont : administration et de la gestion de l'éducation $(26,1 \%)$, de la formation initiale $(18,6 \%)$ et de l'expertise pour les décideurs (6,1\%).

La résolution des problèmes rencontrés en début de carrière est considérée comme une nouvelle année de formation assurée principalement par les supérieurs hiérarchiques $(49,2 \%)$, par des formateurs ou intervenants dans la formation et des collègues de même promotion (27,6 \%) et par l'auto-formation ou recherche-action (23\%). La durée de cette nouvelle formation varie de trois (47,6\%) à six mois (plus de $70 \%$ ).

\section{Discussion}

«L'évaluation à chaud» et même «d'évaluation du show» ou de «pseudo-évaluation » (Vincent, 1990; Soyer, 1998, cités par Ardouin, 2006, p.209) est orientée de manière à faire le marketing de la formation tant à l'intérieur qu'à l'extérieur de la structure de formation, notamment la valorisation des formés. Toutes les promotions issues de cette formation des inspecteurs de l'enseignement perpétuent cette habitude par des cérémonies de remise de diplômes.

La fin de la formation revêt plusieurs significations pour les élèves-inspecteurs. Le succès de fin de formation est la première des satisfactions. Il peut être aussi assorti d'un bon classement car les cinq premiers de chaque promotion peuvent choisir leurs lieux d'affectation. Elle est ensuite synonyme d'un retour auprès de la famille et dans le système éducatif. Enfin, c'est le moment de concrétisation des perceptions, des motivations, de matérialisation des influences reçues. Avec le statut d'inspecteur de l'enseignement naissent une nouvelle confiance en soi et une nouvelle personnalité. Ces sentiments de satisfaction proviennent de la mise en œuvre de compétences dans une situation de travail et de la reconnaissance professionnelle (réassurance et estime de soi) (Jorro, 2007). 
L'évaluation de l'apprentissage aboutit à plusieurs oppositions. La première oppose l'aspect trop théorique et le désintéressement constaté pour le volet pratique, comme si le renforcement académique importait le plus. Dans la formation des inspecteurs, cette situation correspond à une des formes développées par Oudet (2010, p. 87). Il l'appelle alternance organisationnelle, fonctionnelle ou logistique. Elle est sans intention pédagogique, sans volonté de réunir le monde de la formation et le monde du travail, et elle prend la forme d'un calendrier de l'alternance au sens strict du terme. Chaque espace est distinct de l'autre, les espaces s'ignorent. Chacun pilote a sa manière d'effectuer la formation dans une indifférence mutuelle. Même si des objectifs de formation existent de chaque côté, ils n'interagissent pas. Dans cette forme d'alternance, le système de travail et le système formation ont développé des logiques tellement différentes et opposées qu'elles sont devenues contradictoires, au sens où elles se nient l'une et l'autre. C'est cette situation qui prévaut dans la formation des inspecteurs.

La seconde opposition porte sur la relation formateurs-enseignements. Les formateurs sont des inspecteurs titulaires du diplôme universitaire requis à l'entrée en formation d'inspecteur. Le plus souvent, ils ont des diplômes universitaires dits «maîtrise d'enseignement», dans les disciplines utilisées dans l'enseignement secondaire (langues étrangères, philosophie, grammaire, histoire, géographie, etc.), jadis exigé pour accéder à la formation. Après plusieurs années d'exercice dans le système éducatif, ils ont certes acquis de l'expérience, mais celle-ci ne peut remplacer la spécialisation requise pour assurer des enseignements spécialisés (psychologie, sociologie, planification, évaluation, etc.). Ils n’ont donc pas le niveau requis ni la spécialisation attestée pour les enseignements qu'ils assurent. L'absence des vacataires nationaux (consultants, experts, universitaires, chefs de services administratifs, responsables de structures) et d'enseignants étrangers a aussi privé les nouveaux inspecteurs de compétences spécifiques et nécessaires pendant la formation. De même, les absences de spécialistes de l'éducation préscolaire et de l'éducation non formelle justifient aussi l'absence de la polyvalence annoncée dans le plan de formation.

Au total, cette formation a omis «d'intégrer dans un même mouvement l'action au travail, l'analyse de la pratique professionnelle et l'expérimentation de nouvelles façons de travailler»(Wittorski, 2009). Les processus de professionnalisation des individus, des activités et des organisations ne sont pas articulés à une pluralité de modes de professionnalisation.

Dès leur prise de service, les activités principales des nouveaux inspecteurs sont orientées vers les visites de classe et l'animation de cellules pédagogiques. Les chefs de service dont dépend leur emploi de temps les utilisent pour répondre aux besoins d'encadrement des enseignants. Les enseignants en ont besoin pour finaliser leurs examens professionnels, d'autres pour les préparer. L'utilité première de ces interventions est d'améliorer les enseignements et apprentissages. Dans les lieux de travail, les nouveaux inspecteurs ne sont pas toujours sollicités pour les compétences à caractère administratif pour la simple et bonne raison qu'elles sont exclusivement réservées aux responsables de service et à leurs adjoints. Ils les découvrent en fonction de leurs affinités avec les supérieurs hiérarchiques ou en raison de leurs propres initiatives.

La volonté de s'adapter aux changements en cours et d'anticiper les besoins futurs est le résultat attendu de la formation, qui n'est pas atteint. Cette réponse se justifie par les liens de causalité entre les niveaux d'évaluation. Les élèves-inspecteurs avaient un bon niveau d'études et d'expérience et étaient très motivés pour l'apprentissage. Or, le contenu et l'organisation de la formation ne sont pas en phase 
avec les objectifs. Plusieurs compétences ne sont pas acquises (administration, gestion de l'éducation, formation initiale et expertise pour les décideurs). La conséquence immédiate de cette relation entre les apprentissages et les besoins du terrain est que la formation n'est pas en adéquation avec les besoins du terrain.

Ainsi, les connaissances et compétences acquises ne sont pas adaptées à la politique éducative en cours. La formation n'est pas en phase avec les réalités du système éducatif. La formation n'est pas en adéquation avec les besoins du terrain. Plus de $67 \%$ des personnes interrogées ont rencontré de sérieuses difficultés en début de carrière et ont dû suivre et apprendre de nouveau pour s'adapter.

\section{Conclusion}

Au-delà de l'application stricte du modèle de Kirkpatrick qui a établi une hiérarchie entre les étapes et du fait d'entretenir une relation logique, l'évaluation de la formation des inspecteurs s'est appuyée sur les prescriptions d'une évaluation génératrice de développement professionnel. Le déséquilibre dans le déroulement des apprentissages, entre la théorie et la pratique notamment, explique les énormes difficultés rencontrées en début de carrière des inspecteurs. Les compétences acquises et non acquises ont été décelées à la suite d'évaluation des inspecteurs issus de cette formation. Cette évaluation du résultat de la formation s'apparente à une évaluation des compétences. Une autre évaluation identifiée est l'évaluation du dispositif même de formation. Elle porte sur l'organisation pédagogique de la formation, l'environnement de la formation, les acteurs de la formation et les stratégies mises en ouvre. En outre, de par les acteurs qui ont contribué à cette évaluation, plusieurs natures d'évaluation apparaissent. Quand les anciens stagiaires parlent de leurs propres compétences, c'est de l'auto-évaluation. Lorsque les supérieurs hiérarchiques apprécient les produits de la formation, il y a une évaluation externe.

Cette évaluation de formation a permis de mettre en évidence la valeur d'une activité en précisant ses contours positifs, en relevant ses points d'amélioration, en soulignant son intérêt et les questions qui peuvent être dégagées (Jorro, 2000, 2017). Cette évaluation a révélé que la formation n’est pas en phase avec les politiques éducatives en cours, avec les réalités du terrain. Le renforcement de la professionnalisation en est la suggestion principale pour accroître leur efficacité dans le système éducatif.

Cette recommandation renvoie à l'évaluation-conseil dans une approche dialogique (Jorro, 2007). D'une part, en tant qu'interprétation élaborée, elle valorise une action, s'interroge sur des valeurs et distingue des enjeux nouveaux dans une approche dialogique. Dans un autre sens, dans sa visée praxéologique, elle consiste à déclencher des repositionnements, à promouvoir des changements, aussi minimes soient-ils, en tenant compte des contextes d'exercice et des acteurs, notamment de la manière dont ces derniers se projettent dans l'action à venir.

\section{Notes}

1,2 Constats de personnes interrogées sur l'ouverture de la formation à plusieurs intervenants 


\section{Références}

Ardouin, T. (2006). Ingénierie de formation pour l'entreprise : Analyser, concevoir, réaliser, évaluer (2ème édition). Dunod.

Berger, G. (1977). Pour une nouvelle approche de la formation d'inspecteurs. UNESCO.

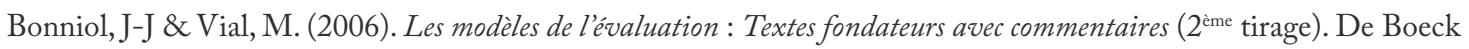

Bourdoncle, R. (1993). La professionnalisation des enseignants : les limites d'un mythe. Revue française de pédagogie, 105, 83-114.

Condette, J.-F. (dir.). (2017). Les personnels d'inspection. Contrôler, évaluer, conseiller les enseignants. Retour sur une histoire, France-Europe (xviie-xxe siècle). PUR

De Ketele, J-M. (2011). Lévaluation et le curriculum : les fondements conceptuels, les débats, les enjeux». Les dossiers des sciences de l'éducation. https://doi.org/10.400/dse.1022

De Ketele,J.-M., \& Gerard, F.-M. (2007). La qualité et le pilotage du système éducatif. Dans M. Behrens, La Qualité en éducation. Pour réfléchir à la formation de demain (pp.19-38). Québec : Presses de l'Université du Québec.

Dickelé, T. (2005). Une évolution des pratiques d'inspection : Chez les inspecteurs de l'Éducation nationale chargés d'une circonscription du premier degré. Informations sociales, 126(6), 74-83. https://doi.org/10.3917/inso.126.0074

Dunberry, A \& Péchard C. (2007). Lévaluation de la formation dans l'entreprise : état de la question et perspectives. CIRDEP. https://www.academia.edu/4209201

Gilibert, D., \& Gillet, I. (2010). Revue des modèles en évaluation de formation : approches conceptuelles individuelles et sociales. Pratiques Psychologiques

Hadji, Ch. (1990). Evaluation, les règles du jeu, ESF.

Jeffrey, D. (2016). L'éthique et la standardisation des pratiques enseignantes. Formation et profession, 24(2), 5-16.http:// dx.doi.org/10.18162/fp.2016.351

Jorro, A. 2000. L'enseignant et l'évaluation. De Boeck.

Jorro, A. (2005). Réflexivité et auto-évaluation dans les pratiques enseignantes. Mesure et Evaluation en Education, Association des spécialistes de la mesure et de l'évaluation en éducation

Jorro, A. (2007). Introduction. L'évaluation-conseil : un processus dialogique au service de la régulation. In Les dossiers des sciences de l'éducation, $\mathrm{N}^{\circ} 18$. L'évaluation-conseil en éducation et formation.

Jorro, A. (2014). Dictionnaire des concepts de la professionnalisation. De Boeck

Jorro, A. (2015). De l'évaluation à la reconnaissance professionnelle en formation. Revue française de pédagogie, 190. https://doi.org/10.4000/rfp.4697

Jorro, A. \& Wittorski, R. (2013). De la professionnalisation à la reconnaissance professionnelle. Les Sciences de l'éducation -Pour l'Ėre nouvelle, vol. 46, nº 4, p.11-22 https://www.cairn.info/revue-les-sciences-de-1-education-pour-1-erenouvelle2013-4-page-11.htm

Oudet, S. (2010). Alternances et professionnalisation. Les dossiers des Sciences de l'Education, 83-95.

Roquet, P. (2012). Comprendre les processus de professionnalisation : une perspective en trois niveaux d'analyse. Phronesis, 1(2), 82-88. https://doi.org/10.7202/1009061

Talbot, L.\& Arrieu-Mutel, A. (2012). Décrire, comprendre et expliquer les pratiques d'enseignement d'un professeur de lycée. Éducation et didactique. https://doi.org/10.4000/educationdidactique.1504

Wittorski, R. (2009). A propos de la professionnalisation. Dans J-M Barbier, E Bourgeois, G Chapelle et JC RuanoBorbalan (dir.). Encyclopédie de l'éducation et de la formation, PUF.

\section{Pour citer cet article}

Balde, I. (2021). Une évaluation de la formation des inspecteurs de l'enseignement au Sénégal. Formation et profession, 29(2), 1-14. http://dx.doi.org/10.18162/fp.2021.598 